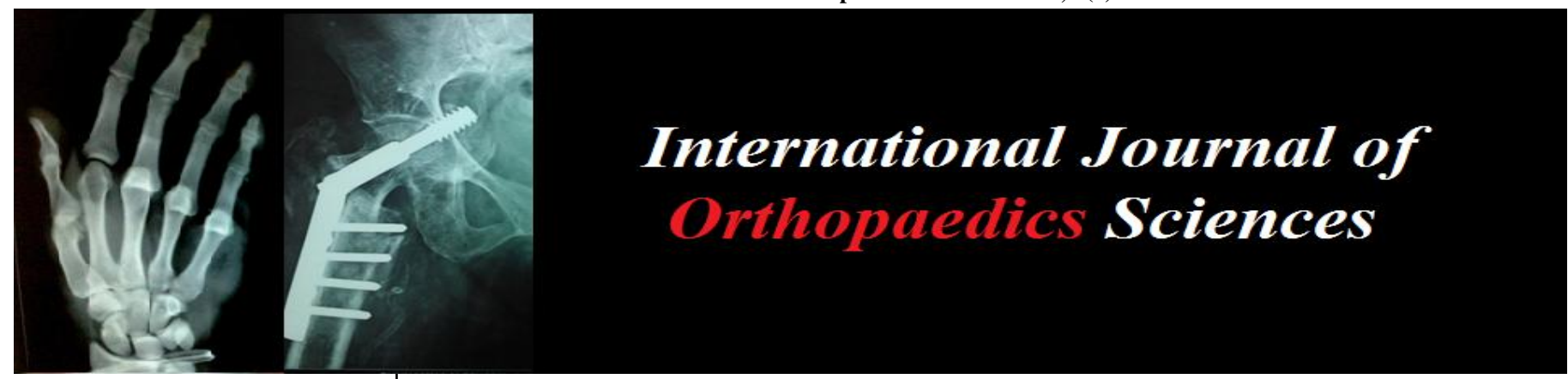

E-ISSN: 2395-1958

P-ISSN: 2706-6630

IJOS 2020; 6(1): 540-544

(C) 2020 IJOS

www.orthopaper.com

Received: 06-11-2019

Accepted: 10-12-2019

Dr. Ashok Meena

SMS Medical College Jaipur, 5 A

Purender Ji Ka Baagh, Mooti

Doongri Road, Jaipur,

Rajasthan, India

Dr. DS Meena

Sms Medical College Jaipur,

Rajasthan, India

Dr. Nirottam Singh

Dr. S.N. Medical College

Jodhpur, Rajasthan, India

Dr. Abhijit SEN

KPC Medical College Kolkata,

West Bengal, India
Corresponding Author:

Dr. Ashok Meena

SMS Medical College Jaipur, 5 A,

Purender Ji Ka Baagh, Mooti

Doongri Road, Jaipur,

Rajasthan, India

\section{Functional outcome of hemi-hamate arthroplasty in proximal interphalangeal joint fracture-dislocations}

\author{
Dr. Ashok Meena, Dr. DS Meena, Dr. Nirottam Singh and Dr. Abhijit SEN
}

DOI: https://doi.org/10.22271/ortho.2020.v6.i1j.1919

\section{Abstract}

Aim of study was to access functional outcome in unstable dorsal PIP joint fracture dislocations. We evaluated 25 patients age between 18 to 35 years. Eligible patient had unstable dislocations with comminuted fractures involving at least $40 \%$ of the volar middle phalangeal surface. Outcomes were assessed by Motion/stability, grip strength and visual analog scales of pain and function. The mean follow-up period was 20 (range 18- 29) months. Surgery performed 38 days after injury (range 8-90 days). Average PIP motion was $88^{\circ}$ range (75-110); DIP motion was $80^{\circ}$ range (75-90), PIP flexion contracture $6.2^{\circ}$ range $\left(0-15^{\circ}\right)$. Hemi-hamate arthroplasty provides good functional outcome with minimal donor site morbidity.

Keywords: PIP dislocation, hamate graft, shotgun volar approach

\section{Introduction}

Proximal interphalangeal joint (PIP) fracture dislocations are complex fracture patterns which are difficult to manage. The PIP joint is more prone to injury due to a long lever arm forces applied to the fingertip. If not treated properly, they can cause pain and poor function with flexion deformities ${ }^{[1]}$. Most accepted classification of the palmar lip injury is given by Hastings and Carroll ${ }^{[2]}$. This classification is based on the percentage of middle phalanx articular surface which is injured. PIP is a highly congruent joint that allow only a single plane of motion. The PIP joint has minimal laxity to compensate for angular, axial or rotational stresses.

Dorsal fracture dislocations are more common than volar dislocations and are considered unstable when the fracture fragment of the middle phalanx base is over $40 \%$ of the articular surface ${ }^{[3]}$. The goal of treatment of any injury around the proximal interphalangeal joint is to establish a congruent joint ${ }^{[4]}$ and allow early mobilization.

A number of surgical options available for the treatment of unstable fractures including external fixation ${ }^{[5,6]}$, volar plate arthroplasty ${ }^{[7,8]}$. Osteochondral autograft 9 and open reduction internal fixation (ORIF) with mini screws and Kirschner wires.

The aim of this study is to determine the clinical and radiological outcome in patients who underwent hemi hamate arthroplasty for PIP dorsal fracture-dislocations.

When $>40 \%$ of the PIP joint surface is fractured there is dorsal PIP subluxation occur because the entire volar bony buttress is lost. Collaterals are attached only to the fracture fragment which creates unstability to PIP joint.

\section{Materials and Methods}

This Prospective study was carried out in department of Orthopaedics, SMS Medical College \& Hospital, Jaipur from April 2016 to December 2018. 25 patients were included in the study with unstable dorsal proximal interphalangeal fracture dislocations. (Joint comminuition of $\geq$ $40 \%)$.

Mean age was 24.7 years (range 18-35). Most patients were students $68.0 \%$, followed by teacher $8.0 \%$. Most common mode of injury was Sports injury (19/25) due to cricket ball followed by RTA (4/25) and door entrapment (2/25). 


\section{Clinical examination \& Surgical Technique}

Inspection of the finger, for any swelling and tenderness. Radiograph including both true anterior-posterior and lateral view should be done and document dorsal "V sign for instability in true lateral view.

We used radially based volar flap to Access the Pip joint, incision was given from the base of the proximal digital crease to the distal interphalangeal (DIP) joint crease (Fig.1). Dissection done superficial to the neurovascular bundle down to the flexor sheath, elevating the subcutaneous tissue off the sheath and retract the neurovascular bundles. The sheath was incised between the A2 and A4 pulleys along the lateral edge. The flexor tendons are retracted. The accessory collaterals insert into the volar plate which were incised longitudinally. The volar plate is divided transversely at its distal insertion and elevated to its proximal insertion. The collateral ligaments released from their proximal insertion. Joint is bent dorsally $150^{\circ}$ (shotgun- approach) and the flexor tendons were carefully retracted to the radial or ulnar side, the volar plate was folded proximally (Fig. 2, 3). At this point, the fracture site was completely exposed.

In chronic cases, a elevator is required to free the dorsal capsule and adhesions. The volar fragments were excised and prepared smoothly to facilitate firm placement of the hemihamate autograft. After measuring the bony defect for the appropriate graft size (Fig.4).We returned the PIP joint to neutral position.

Next, incision is made over the base of the fourth and fifth metacarpals. The capsule was incised and elevated to expose the hamate. Hamate has a central ridge and bicondylar facet with articular contours which are similar to the base of the middle phalanx. The appropriate size donor graft measured. The proximal end of the graft should be thicker than the articular portion to allow the graft to recreate the volar lip and appropriate buttress. The graft is prepared after removal to fit in the defect (Fig.5). The graft is held in place with a provisional $1 \mathrm{~mm}$ Kirschner wire, and then two $1.3-\mathrm{mm}$ screws are placed in lag fashion to secure the graft. (Fig.6) to the intact dorsal cortex to prevent movement. After the joint was reduced (Fig.7), graft position \& size of screw confirmed by X-Ray. The volar plate was reattached to the fibrous remnant of the accessory collateral ligaments from which it was detached. Joint stability checked in flexion and extension position. We repaired the flexor sheath in its anatomical position.

\section{Postoperative rehabilitation protocol}

After operation, a dorsal splint was applied with PIP joint in $20^{\circ}$ flexion to protect the volar plate. On next day of surgery, patients were allowed to perform PIP mobilization and passive stretching exercises without hyperextension. After 2 weeks full active and passive ROM exercises were started with dorsal splint on fingers to maintain the PIP joint in neutral extension.

\section{Follow up}

Clinical postoperative evaluation done that includes measurement of the range of motion at the proximal inter phalangeal, and distal inter-phalangeal joints. Extension deficits of the PIP joint was noted. Grip strength was obtained for the injured and non-injured hand. Antero-posterior and lateral radiographs were taken at 4 to 6 weeks to evaluate bone healing and joint congruity.

\section{Statistical analysis}

Descriptive statistics of injury and surgery characteristics were performed.

\section{Results}

A total of 25 patients were included the study, 24 were male and 1 was female. The mean age was 24.7 years (range 18 35 ). The mean follow-up period was 20 months (range 18-29 month).

Radiological evaluation confirmed union of the graft in all patients after 5.9 weeks (range 5-7).

(Figure 10). Delay between injury and operation on average was 38 days (range 0-90). Mean operation time was $90 \mathrm{~min}$ (range 75-120). Power grip strength in the operated hand was $91 \%$ of the healthy side. Total range of motion (ROM) of the PIP joint was $88^{\circ}$ (range 75-110).

Extension deficit of the PIP joint on average was $6.2^{\circ}$ (range 0-15), ROM of the DIP joint was $75^{\circ}$ (range 75-90). (Fig. $10,11)$. In our study Post operative pain, ability to perform daily Activities. were assessed. 21 patients reported no pain associated with the injured finger and remaining 4 patients reported pain only with heavy activity. All patients were return to their previous occupation.

\section{Complication}

There was no infection and one patient had revision surgery due to loosening of screw during mobilization. All patients were satisfied and no residual pain at involved digit Except one patient who had mild pain and unable to completely straighten the finger.

\section{Discussion}

Most of the PIP joint fracture dislocations are dorsal dislocation and associated with fracture of the volar articular surface of the base of middle phalanx. Stable injuries are treated conservatively. For unstable acute injuries $(<6$ week $)$ various treatments has been proposed including extension block Orthosis, percutaneous pinning, external fixation5,6, traction methods ${ }^{[10]}$, static and dynamic, open reduction internal fixation(ORIF) with mini screw, volar plate

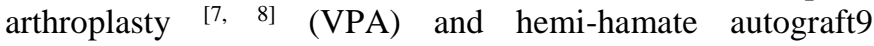
reconstruction.

Chronic unstable injuries are difficult to manage and may leads to persist pain, stiffness and disability. Treatment options for these injuries are limited that includes Osteotomy with open reduction and internal fixation, PIP joint arthrodesis or arthroplasty, Volar plate arthroplasty ${ }^{[7,8]}$. and hemi-hamate arthroplasty9. Volar plate arthroplasty causes recurrent dorsal subluxation, angulation, decrease PIP motion, distal interphalangeal (DIP) joint stiffness. Internal fixation with mini-screws provide several advantages over the K-wires but with multiple fracture fragments that are difficult to hold properly. With External fixation devices (Suzuki fram) there was risk of pin tract infections that are beneficial in fresh injury.

The concept of using an osteochondral autograft was advanced by Hastings et al. ${ }^{[11]}$ in 1999 , who described the use of a hemi-hamate autograft for the treatment of PIP fracturedislocations. The main goal of this technique in restoration of both a congruent articular surface. N. Lindenblatt et al. ${ }^{[12]}$ studied Hemi hamate Arthroplasty for acute and chronic PIP Joint Fracture Dislocations. After 93 days (range 0-371 days) of injury, average PIP motion was $71^{\circ}$ (range 0-90); DIP motion was $54^{\circ}$ (range $10-90$ ) with a mean PIP flexion contracture of $6.5^{\circ}$ (range 0-20). Grip strength was average 
$91 \%$ of opposite hand. M. Burnier et al. ${ }^{[14]}$ in 2016 , found the mean active flexion at the proximal interphalangeal joint was to $83^{\circ}$ with a mean fixed flexion of $17^{\circ}$. The mean active distal interphalangeal motion was $41^{\circ}$.

We treated 25 patients in our hand surgery unit with this technique. Re-established range of motion of the PIP joint averaged $88^{\circ}$ including an average PIP extension deficit of $6.2^{\circ}$. In our study we found that results are better, the difference may be due to early surgical intervention and early mobilization of PIP joint that helps to maximize ROM in the injured digit and prevent adhesions.

Study by Williams et al. showed that the capability of this technique to re-establish the volar base of the middle phalanx which provides joint stability and early motion rehabilitation [13]. Williams et al. in 2003, found a total range of motion of the PIP joint of $85^{\circ}$ with an average extension deficit of $9^{\circ}$ was almost similar to our study. The average follow-up period was shorter in our study as compared to the Williams et al., (8 vs. 16 months). The mean time between injury and hemihamate arthroplasty in their study was 45 days and in our study it was 38 days which indicate there were acute injuries with less long-term consequences like collateral band shortening, volar plate adhesions, joint capsule stiffness, etc. Grip strength was $91 \%$ of the uninjured hand after the operation.

The short-term results of hamate osteochondral grafts for PIP fracture-dislocation are good, longe term assessments must be required to for cartilage viability.

Studies evaluating long-term results with respect to the development of secondary osteoarthritis after hemi-hamate arthroplasty reported that the development of mild to severe osteoarthritis is roughly in $50 \%$ of the cases ${ }^{[13]}$. The followup in the our study was not long enough to account for these long-term sequelae.

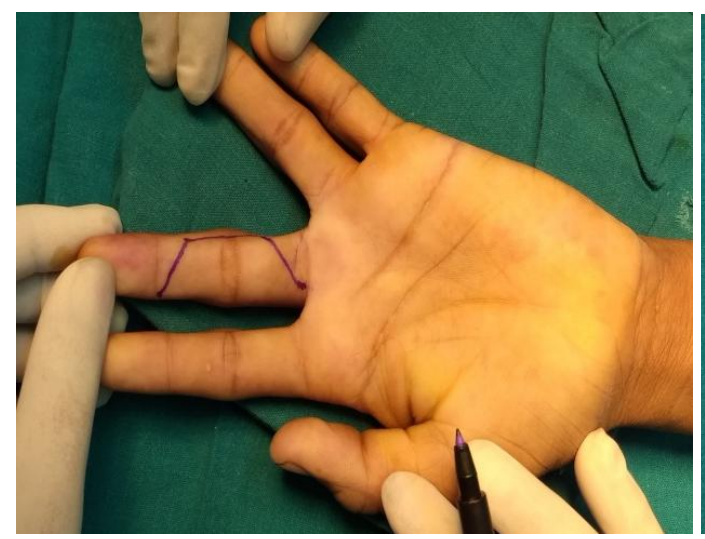

Fig 1: Access PIP- via a palmar incision

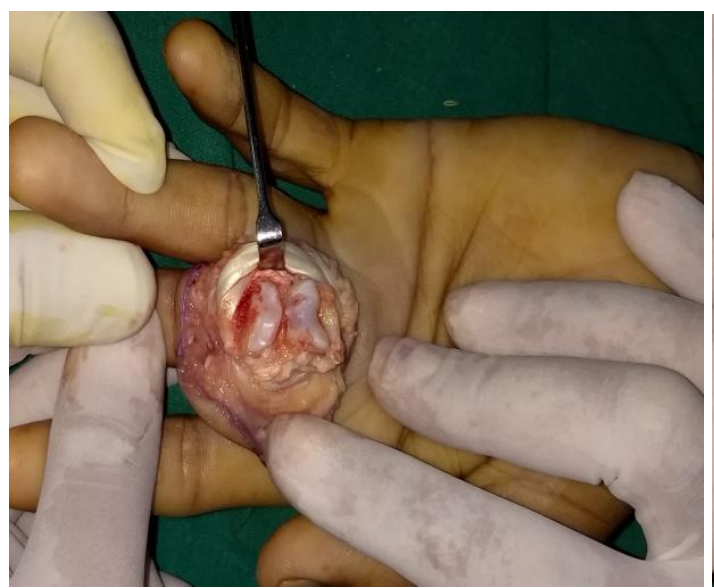

Fig 3: Joint is bent dorsally $150^{\circ}$ (shotgun approach) and the joint surface, Fracture site becomes visible.

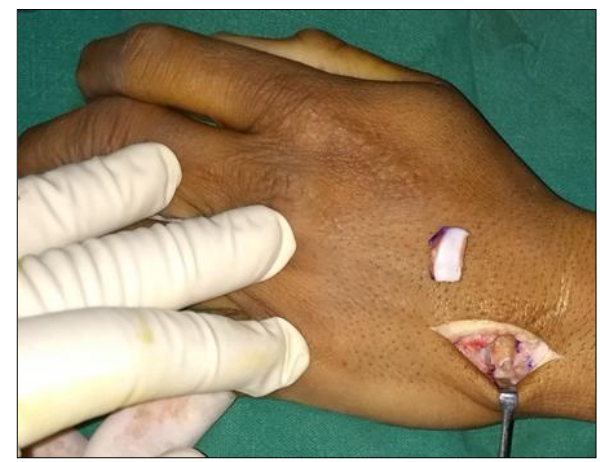

Fig 5: Graft is harvested from the dorsum of the hamate.

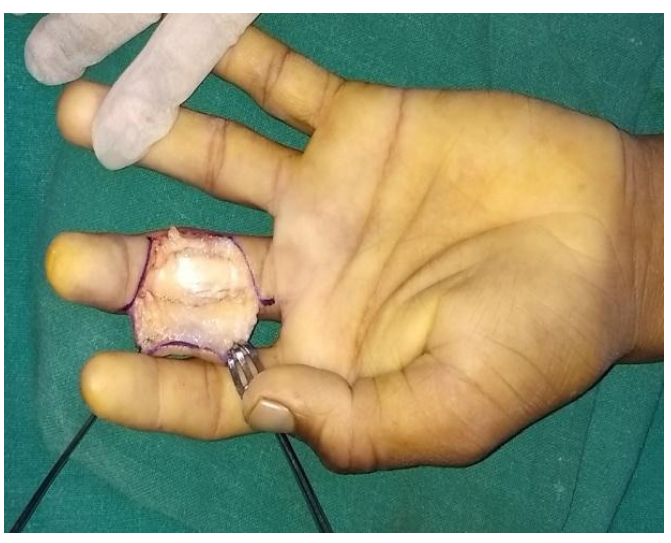

Fig. 2

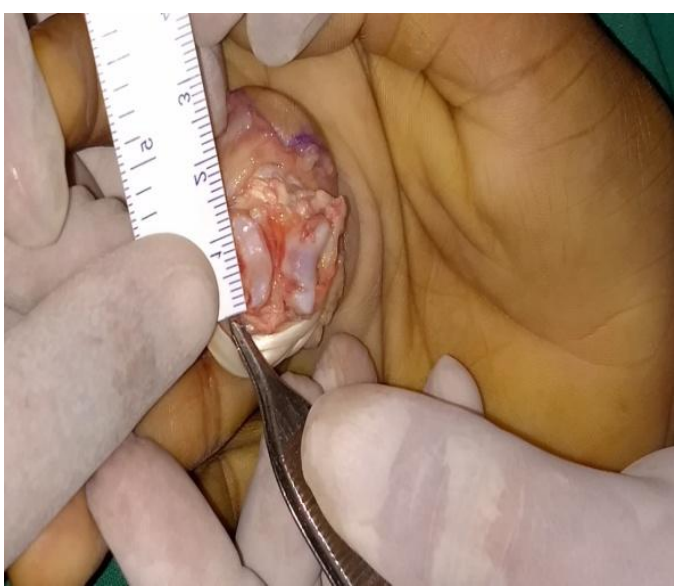

Fig 4: Dimensions of the bony defect are measured

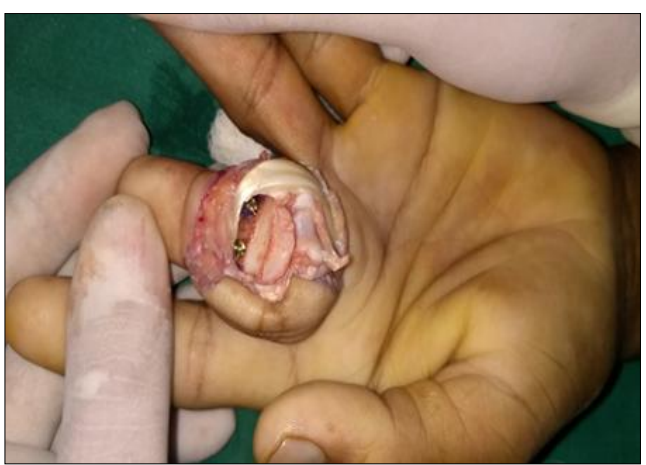

Fig 6: Hamate autograft in place and screws 


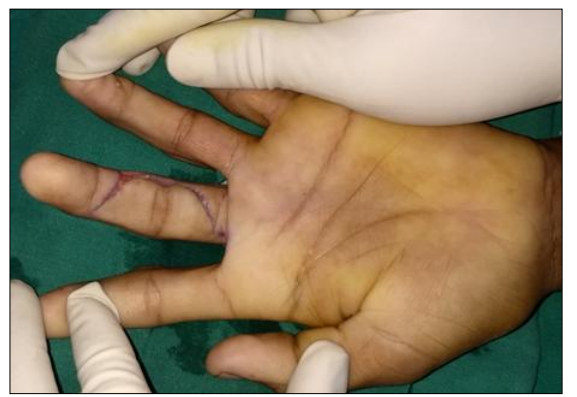

Fig.7 Reduction joint after Graft

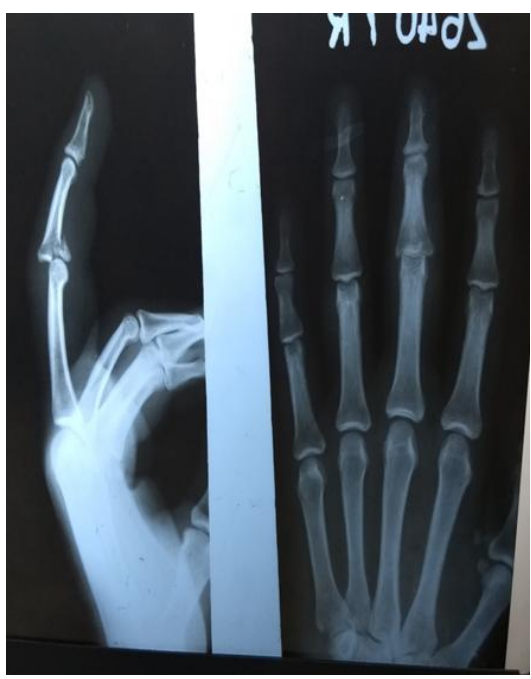

Fig 8: Pre op. X-Ray

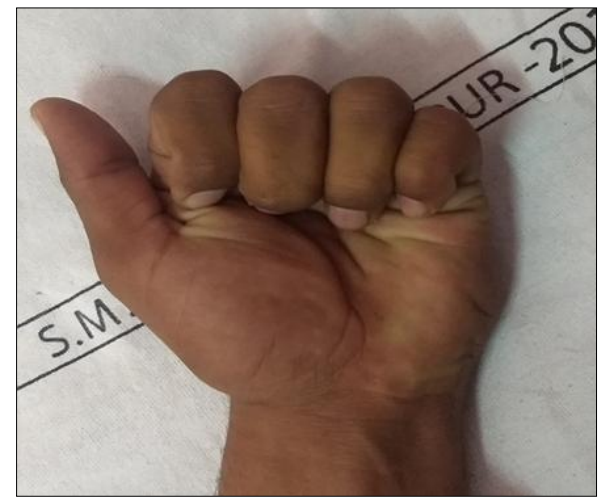

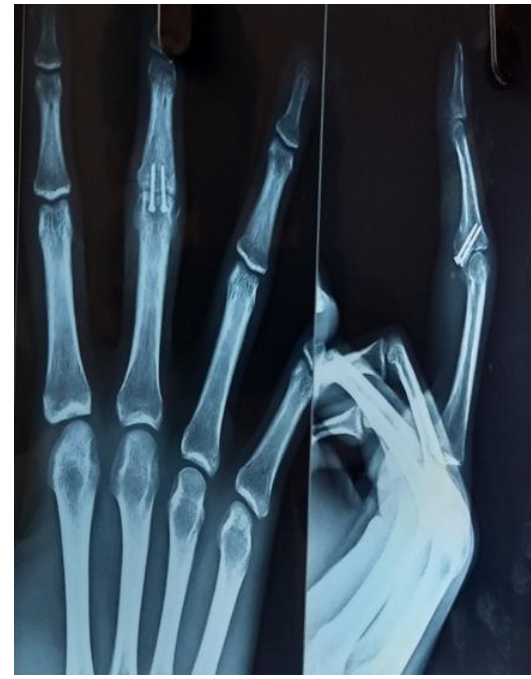

Fig 9: Post op. (5 week)-complete graft union

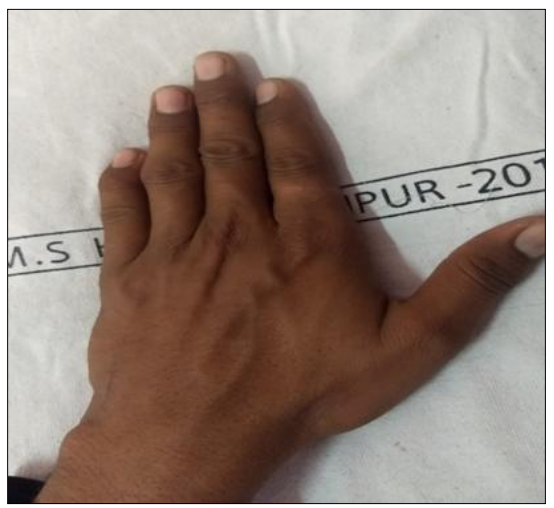

Fig 10-11: Post. operative hand function following hemi-hamate arthroplasty of the pip joint of the middle finger

Table 1: Patient characteristics

\begin{tabular}{|c|c|c|c|c|c|c|}
\hline $\begin{array}{c}\text { S. } \\
\text { No }\end{array}$ & Age & Involved Hand & Injured Finger & Mode of Injury & $\begin{array}{c}\text { Time Between Injury } \\
\text { And Surgery (Days) }\end{array}$ & $\begin{array}{c}\text { \% Base Middle } \\
\text { Phalanx Fracture }\end{array}$ \\
\hline 1 & 18 & Dominant & Middle Finger & sports injury (cricket ball) & 90 & $40 \%$ \\
\hline 2 & 24 & non dominent & Ring finger & door Entrapment injury & 45 & $50 \%$ \\
\hline 3 & 35 & Dominant & Middle Finger & RTA & 30 & $50 \%$ \\
\hline 4 & 20 & Dominant & Ring finger & sports injury (cricket ball) & 35 & $40 \%$ \\
\hline 5 & 18 & Dominant & Middle Finger & sports injury (cricket ball) & 60 & $40 \%$ \\
\hline 6 & 25 & Dominant & Middle Finger & sports injury (cricket ball) & 25 & $50 \%$ \\
\hline 7 & 18 & non dominent & Ring finger & sports injury (cricket ball) & 25 & $40 \%$ \\
\hline 8 & 21 & Dominant & Ring finger & sports injury (cricket ball) & 55 & $40 \%$ \\
\hline 9 & 22 & nondominent & Middle Finger & sports injury (cricket ball) & 8 & $50 \%$ \\
\hline \hline 10 & 32 & Dominant & Middle Finger & RTA & 50 & $50 \%$ \\
\hline 11 & 21 & nondominent & Ring finger & sports injury (cricket ball) & 22 & $50 \%$ \\
\hline 12 & 20 & Dominant & Middle Finger & sports injury (cricket ball) & 40 & $40 \%$ \\
\hline 13 & 26 & Dominant & Ring finger & sports injury (cricket ball) & 30 & $40 \%$ \\
\hline 14 & 22 & Dominant & Middle Finger & sports injury (cricket ball) & 70 & $50 \%$ \\
\hline 15 & 25 & nondominent & Middle Finger & sports injury (cricket ball) & 35 & $40 \%$ \\
\hline 16 & 32 & Dominant & Ring finger & RTA & 22 & $60 \%$ \\
\hline 17 & 25 & Dominant & Middle Finger & door Entrapment injury & 15 & $50 \%$ \\
\hline
\end{tabular}




\begin{tabular}{|l|l|c|c|c|c|c|}
\hline 18 & 24 & non dominent & Ring finger & sports injury (cricket ball) & 37 & $50 \%$ \\
\hline 19 & 20 & Dominant & Ring finger & sports injury (cricket ball) & 45 & $60 \%$ \\
\hline 20 & 28 & nondominent & Middle Finger & sports injury (cricket ball) & 30 & $50 \%$ \\
\hline 21 & 26 & nondominent & index finger & sports injury (cricket ball) & 45 & $60 \%$ \\
\hline 22 & 27 & nondominent & Ring finger & sports injury (cricket ball) & 30 & $60 \%$ \\
\hline 23 & 30 & nondominent & Ring finger & sports injury (cricket ball) & 40 & $50 \%$ \\
\hline 24 & 33 & Dominant & Middle Finger & RTA & 30 & $40 \%$ \\
\hline
\end{tabular}

Table 2: Outcome of hemi-hamate arthroplasty

\begin{tabular}{|c|c|c|}
\hline PIP ROM deg. (Post Op.) & Flexion Deformity at PIPJ & Radiological Union (WK) \\
\hline 100 & 5 & 6 \\
\hline 85 & 10 & 6 \\
\hline 80 & 10 & 5 \\
\hline 95 & 0 & 6 \\
\hline 85 & 10 & 7 \\
\hline 80 & 10 & 6 \\
\hline 110 & 0 & 5 \\
\hline 80 & 5 & 7 \\
\hline 90 & 10 & 6 \\
\hline 85 & 5 & 5 \\
\hline 95 & 0 & 6 \\
\hline 95 & 0 & 6 \\
\hline 80 & 15 & 5 \\
\hline 80 & 10 & 6 \\
\hline 85 & 5 & 7 \\
\hline 80 & 10 & 6 \\
\hline 85 & 5 & 5 \\
\hline 80 & 0 & 6 \\
\hline 90 & 5 & 7 \\
\hline 90 & 10 & 6 \\
\hline 95 & 5 & 6 \\
\hline 80 & 10 & 7 \\
\hline 90 & 5 & 6 \\
\hline 95 & 5 & \\
\hline 90 & 5 & \\
\hline & & \\
\hline
\end{tabular}

\section{Conclusion}

Hemi-hamate arthroplasty is an excellent procedure for unstable dorsal fracture dislocations of PIP Joint with minimal donor site morbidity. Hemi-hamate arthroplasty restores a smooth joint surface of base of the middle phalanx.

Financial support and Sponsorship: Nil.

Conflicts of interest: No conflicts of interest.

\section{References}

1. Benke GJ, Stapleforth PG. Injuries of the proximal interphalangeal joint of the fingers. Hand. 1979; 11:263.

2. Hastings H, Carroll C. Treatment of closed articular fractures of the metacarpophalangeal and proximal interphalangeal joints Hand Clin. 1988; 4:503-527.

3. Kiefhaber TR, Stern PJ. Fracture dislocations of the proximal interphalangeal joint. J Hand Surg Am. 1998; 23(3):368-80.

4. Wilson JN, Rowland SA. Fracture-dislocation of the proximal interphalangeal joint of the finger. J Bone Joint Surg Am. 1996; 48:493-502.

5. Hastings HII, Ernst JM. Dynamic external fixation for fractures of the proximal interphalangeal joint. Hand Clin. 1993; 9:659-674.

6. Agee JM. Unstable fracture dislocations of the proximal interphalangeal joint. Treatment with the force couple splint. Clin Orthop. 1987; 214:101-112.

7. Deitch MA, Kiefhaber TR, Comisar BR, Stern PJ. Dorsal fracture dislocations of the proximal interphalangeal joint: surgical complicationsn and long-term results. J
Hand Surg Am. 1999; 24(5):914e-923.

8. Dionysian E, Eaton RG. The long-term outcome of volar plate arthroplasty of the proximal interphalangeal joint. J Hand Surg Am. 2000, 25.

9. Williams RM, Kiefhaber TR, Sommerkamp TG et al. Treatment of unstable dorsal proximal interphalangeal fracture dislocations using a hemi-hamate autograft. J Hand Surg Am. 2003; 28:856-865, (3):429e437.

10. Schenck RR. Dynamic traction and early passive movement for fractures of the proximal interphalangeal joint. J Hand Surg. 1986; 11A:850-858.

11. Hastings H, Capo J, Steinberg B et al. Hemicondylar hamate replacement arthroplasty for proximal interphalangeal joint fracture/dislocations. 54th Annual Meeting of the American Society for Surgery of the Hand. Boston MA, 1999.

12. Lindenblatt N, Biraima A, Tami I, Giovanoli P, Calcagni M. Hemi-hamate Autograft Arthroplasty for Acute and Chronic PIP Joint Fracture Dislocations. \{Handchir Mikrochir Plast Chir. 2013:45:13-19.

13. Afendras G, Abramo A, Mrkonjic A, Geijer M, Kopylov P, Ta* Gil M. Hemi-Hamate Osteochondral Transplantation In Proximal Interplalangeal Dorsal Fracture Dislocations. (The Journal of Hand Surgery, European. 2010; 35E(8):627-631.

14. Burnier M, Awada T, Marin Braun F, Rostoucher P, Ninou M, Erhard L. Unstable proximal interphalangeal joint fractures with hemi-hamate osteochondral autografts Hemihamate arthroplasties The Journal of Hand Surgery, 2016 . 